\title{
Germanica
}

\section{De l'éducation corruptrice ou : Les années de déformation du jeune Kafka}

Von der schädlichen Erziehung oder : Franz Kafkas "Verbildungsjahre"

\section{Florence Bancaud}

\section{(2) OpenEdition}

\section{Journals}

Édition électronique

URL : http://journals.openedition.org/germanica/2152

DOI : $10.4000 /$ germanica.2152

ISSN : 2107-0784

Éditeur

Université de Lille

Édition imprimée

Date de publication : 30 juin 2002

ISBN : 9782913857070

ISSN : 0984-2632

Référence électronique

Florence Bancaud, « De l'éducation corruptrice ou : Les années de déformation du jeune Kafka », Germanica [En ligne], 30 | 2002, mis en ligne le 16 juillet 2013, consulté le 06 octobre 2020. URL : http://journals.openedition.org/germanica/2152; DOI : https://doi.org/10.4000/germanica.2152

Ce document a été généré automatiquement le 6 octobre 2020.

(C) Tous droits réservés 


\title{
De l'éducation corruptrice ou : Les années de déformation du jeune Kafka
}

\author{
Von der schädlichen Erziehung oder : Franz Kafkas « Verbildungsjahre »
}

\author{
Florence Bancaud
}

\begin{abstract}
Une de nos erreurs indéracinables consiste à mésestimer la jeunesse en y voyant une phase de la vie à laquelle la plupart des valeurs existentielles telles que le bonheur, la liberté, la beauté, la faculté à éprouver des sensations fortes sont inhérentes. Rien ne correspond moins à la réalité. [...] La jeunesse n'est pas seulement une période de contrainte quasi intolérable, de répression et de tutelle constamment imposée de l'extérieur [...]; elle est en soi une maladie qui demande à être surmontée [...]; on ne se possède pas plus que l'on ne possède le monde, tout le temps que dure la jeunesse; on vit dans une misère innommable parce que l'on ne peut donner le nom juste ni à soi-même ni aux choses du monde alentour ${ }^{1}$.
\end{abstract}

1 Franz Kafka, né en 1883, au tournant du siècle, a dix-sept ans en 1900. Il assiste alors à l'éclosion de l'Art nouveau qui prétend rompre avec un historicisme et un formalisme sclérosés et renouveler la perception des formes en préférant à la ligne et l'angle droit la courbe végétale, reflet des permanentes mutations de la nature, du dynamisme de la vie et de la sensualité des êtres et des choses. Le mythe de la jeunesse est alors à son comble et l'on pourrait donc s'attendre à ce que Kafka, observateur si attentif de son temps, se fasse l'écho de ce culte de la vie intense et de l'énergie juvénile.

2 Or, lorsque l'on regarde les portraits des enfants Kafka, si l'on lit une certaine sérénité sur le visage de ses trois sœurs, on est frappé par la gravité du regard et la rigidité de l'attitude du jeune Franz. Une simple photographie suffit à traduire la profonde solitude de cet être qui paraît absent, perdu dans ses réflexions et ses rêves. Dès sa plus tendre enfance, Kafka porte en effet sur la vie le regard d'un adulte, comme s'il avait grandi trop vite.

3 Le thème de l'enfance et de la jeunesse est donc d'autant plus intéressant qu'il paraît quasi absent, comme refoulé de l'œuvre narrative. Kafka ne met pour ainsi dire jamais d'enfants en scène dans ses romans. Les jeunes filles y apparaissent très fugitivement, 
et les personnages de jeunes hommes sont rares et toujours célibataires. Dans le Journal, chaque fois qu'il évoque son enfance et sa jeunesse, c'est pour signifier qu'elles ont été gâchées, « corrompues » selon ses propres termes.

4 L'enfance est traditionnellement le prélude à l'âge adulte et représente une sorte de paradis perdu, de monde de fraîcheur et de naïveté, protégé par l'amour parental et égayé par les jeux avec la fratrie existante. Or, chez Kafka, elle semble n'avoir jamais existé et incarne donc pour l'adulte soit une période maudite et donc refoulée, soit un rêve impossible.

Quant à la jeunesse, qui représente généralement une phase d'émancipation de la tutelle parentale, d'éveil des sens, d'identification de ses désirs et ses attentes par rapport à l'existence, puis d'adaptation progressive au monde social, elle fait seulement office chez Kafka, condamné à l'«hésitation devant la naissance", de phase intermédiaire entre une enfance manquée et un âge adulte avorté. Ainsi s'explique le paradoxe selon lequel Kafka, sans avoir jamais vécu d'enfance, est resté un éternel enfant, à jamais nostalgique de la jeunesse qui lui a été refusée et est mort à quarante et un ans, "précocement vieux", sans s'être véritablement confronté au chaos qu'il désirait autant qu'il le redoutait : «Absurdité de la jeunesse. Peur de la jeunesse, peur de l'absurdité, de la croissance absurde de cette vie inhumaine $»^{2}$.

\section{L'enfance et la jeunesse de Franz Kafka}

6 Franz Kafka naît le 3 juillet 1883, dans un quartier de Prague à la limite entre les beaux quartiers et le ghetto. Il est l'aîné de six enfants. Les deux garçons qui lui succèdent, Georg, en 1885 et Heinrich en 1887, meurent en bas âge. Puis naissent trois filles : Elli, en 1889, Valli en 1890 et Ottla en 1892. La petite enfance de Kafka est solitaire et angoissée :

J'étais un enfant craintif, ce qui ne m'empêchait pas d'être têtu, comme le sont les enfants ; il est certain aussi que ma mère me gâtait, mais je ne puis croire que j'aie été un enfant particulièrement difficile à mener, je ne puis pas croire qu'on n'eût pu obtenir tout ce qu'on voulait de moi en me parlant sur un ton affectueux, en me prenant posément la main, en me regardant avec bonté3.

7 Le jeune Franz reste le seul enfant mâle de sa famille, nimbée du fantôme de ses deux frères morts, et est déjà âgé de six ans lorsque naît sa sœur Elli. Les déménagements des parents sont fréquents et engendrent un climat quelque peu instable. En 1889, lorsqu'Hermann Kafka achète la belle maison de l'Altstädter Ring où il installe son épicerie, l'éducation de Franz est confiée à des bonnes, cuisinières ou gouvernantes. L'autorité paternelle ne se manifeste que négativement; Hermann est tyrannique et conçoit l'éducation comme une discipline à laquelle l'enfant doit se soumettre avec obéissance et résignation :

De ton fauteuil tu gouvernais le monde. Ton opinion était juste, toute autre était folle, extravagante, meschugge, anormale [...]. Pour l'enfant que j'étais, tout ce que tu me criais était positivement un commandement du ciel, je ne l'oubliais jamais, cela restait pour moi le moyen le plus important dont je disposais pour juger le monde ${ }^{4}$.

Décidé à forger la personnalité de ses enfants plutôt qu'à la laisser s'épanouir librement, Hermann veut faire du jeune Franz, timide, chétif, taciturne et contemplatif, un être fort, actif et tonitruant, à son image. Quant à la mère, elle reste très passive ou cherche à s'interposer entre son époux et ses enfants, auxquels elle ne donne que peu 
d'affection, et avec qui elle ne communique que très peu, tant elle souffre de la tension du climat familial.

De cette petite enfance instable et dénuée d'affection parentale, Kafka hérite le sentiment d'une profonde solitude et d'une grande frustration affective, qu'il tente de compenser en observant son entourage, en se faisant, comme il l'écrira plus tard dans son Journal, le « contemplateur à mi-distance de la vie ». Il parvient aussi à surmonter les pesanteurs familiales en s'évadant dans le monde du sport, de l'humour et de la littérature et en devenant peu à peu le mentor de ses sœurs. Dès 1896, dans la maison de la Zeltnergasse, il écrit et met en scène de petites pièces de théâtre qu'il joue avec ses sœurs devant ses parents, et, attentif à sa propre santé, les incite à nager, faire du bateau et du ski.

Les trois sœurs Kafka ne souffrent pas, comme Franz, de cette solitude et de cet écart avec la communauté familiale : elles sont considérées comme une entité et sont élevées comme telles par des bonnes, des gouvernantes ou des préceptrices qui les accompagnent partout et soumettent à un contrôle permanent de leurs dires, faits et gestes. Comme dans le cas de Franz, l'éducation n'a nullement pour but d'épanouir leurs aptitudes et de satisfaire leurs désirs individuels, mais de faire d'elles des épouses et des mères accomplies.

11 Franz, isolé jusque dans l'éducation spéciale qui lui est dispensée et qui lui donnera cependant une certaine autorité sur ses sœurs, entre à six ans à l'école primaire allemande et en sort à dix ans pour faire ses humanités dans un lycée classique allemand, pour satisfaire à l'assimilationisme et aux ambitions sociales de son père. $\mathrm{Ce}$ dernier veut élever son fils dans la langue allemande, celle des classes dirigeantes, qui ouvre la voie aux carrières libérales et administratives. L'enseignement qui est dispensé, l'étude des langues, de la littérature allemande et des civilisations grecque et latine, est austère et la discipline étouffante. Toutefois, Franz parvient à se lier d'amitié à Oskar Pollak et, dès 1897-1898, à quatorze ans, écrit ses premiers récits.

Après son baccalauréat en 1901, Franz s'inscrit à l'université de Prague, où il éprouve la plus grande difficulté à trouver sa voie. Il se lasse vite des études de chimie, puis de droit romain, et opte pour des études littéraires, mais les cours de littérature allemande prodigués par Auguste Sauer le déçoivent. Il songe, avec son ami Pollak, à quitter Prague pour Munich, puis y renonce pour rester à Prague. Il interrompt alors ses études littéraires pour se consacrer, sans enthousiasme et par devoir, au droit, pour lequel il obtiendra un doctorat en 1906. À l'université, il mène une vie intellectuelle et culturelle intense, allant au théâtre, aux conférences de divers cercles dont le cercle culturel des étudiants allemands où il rencontre celui qui deviendra son fidèle ami, Max Brod, et assiste notamment aux discussions des disciples du philosophe Brentano au cercle du Louvre. Pendant ces années d'université, l'activité littéraire de Kafka est intense, mais ses écrits de jeunesse et d'adolescence seront tous détruits, comme si Franz reniait sa jeunesse comme une période hybride. Les premiers écrits conservés sont Description d'un combat (1904-1907) et Préparatifs de noce à la campagne (1907) : Kafka est alors âgé de vingt et un ans et il ne reste nulle trace de ses œuvres d'adolescent. Cette période où il fait de nombreux projets d'avenir lui garantissant la possibilité d'écrire loin de Prague et de sa famille et où il envisage même d'émigrer en Amérique, se clôt lorsqu'il se condamne, en acceptant de travailler comme employé aux Assicurazioni generali, puis à l'assurance ouvrière contre les accidents du travail, à rester à Prague, à subir les 
pesanteurs bureaucratiques et donc à renoncer précocement à la liberté physique et morale dont il avait rêvé.

\section{Le problème du père}

13 C'est le conflit avec le père qui rapproche Kafka des écrivains expressionnistes nés pour la plupart entre 1890 et 1895 , nourris de la lecture de Nietzsche et liés par un vif sentiments de solidarité et d'hostilité envers le monde des adultes et la tradition. C'est une génération hantée par le mythe de l'énergie juvénile et la rupture avec les tables de la loi écrites par les pères, comme en témoigne la floraison de revues expressionnistes telle la revue des Weiße Blätter de Kurt Wolff, éditeur et ami de Kafka. Ces revues permettent aux jeunes écrivains d'alors d'exprimer leurs espoirs et utopies et de prendre conscience de leurs intérêts communs tout en luttant contre un monde sclérosé par les adultes. Beaucoup d'œuvres expressionnistes sont ainsi consacrées au conflit père-fils et à l'affirmation du droit de la jeunesse à vivre sa vie et à lutter contre un système d'éducation rigide que l'écrivain pragois Ernst Weiss appelle les « fabriques de l'amour et de l'esprit ».

14 C'est là le problème central auquel les quatre enfants Kafka ont été, dès leur plus jeune âge, confrontés, tentant désespérément de trouver un modus vivendi avec un père écrasant et une mère impuissante. Les trois filles y sont toutefois mieux parvenues que Franz.

15 Des trois filles, c'est Elli qui eut le plus de difficultés avec son père. À sa naissance, Hermann Kafka espérait un deuxième garçon et n'a donc cessé de la poursuivre de sa rancœur, de l'insulter et de la contraindre par exemple à s'asseoir à dix mètres de la table pendant les repas :

C'était pour moi une vraie fête de la méchanceté et de la joie maligne que de t'entendre lui dire presque à chaque repas quelque chose comme : « il faut qu'elle se tienne à dix mètres de la table, cette grosse donzelle » et de te voir, plein de colère dans ton fauteuil, essayer d'imiter sans la plus légère trace de gentillesse ou de bonne humeur, en ennemi acharné, la manière exécrable selon toi dont elle se tenait à table .

16 Mais Elli parviendra à s'accommoder de cette défaveur et, toute sa vie durant, elle évitera les conflits et gardera ses distances par rapport à son père. Elle partage avec sa sœur Valli une grande passivité explicable par le refus que le père oppose à toute activité que ses filles entreprennent. Une grande solidarité unit les trois sœurs dans leur prévention contre le père. C'est d'ailleurs Valli, la sœur cadette, qui entretiendra les rapports les moins problématiques avec Hermann Kafka. Quant à Franz, il se sent en permanence inférieur à son père ; il est pris dans une fatale dialectique entre le désir de lui ressembler pour être reconnu de lui et la culpabilité de ne pas le pouvoir :

Ce sentiment de mon néant qui s'empare si souvent de moi [...] tient pour beaucoup à ton influence. Il m'aurait fallu un peu d'encouragement, un peu de gentillesse, j'aurais eu besoin qu'on dégageât un peu mon chemin, au lieu de quoi tu me le bouchais, dans l'intention, louable, certes, de m'en faire prendre un autre ${ }^{6}$.

17 Il se trouve déchiré entre le mépris de lui-même et la haine d'un père incapable de détecter sa sensibilité littéraire et contre la médiocrité duquel il tente de lutter en élevant le niveau culturel de sa famille par des conseils de lecture et de pièces de théâtre destinés à réduire l'écart intellectuel qui le sépare de ses proches. 
18 Toute leur enfance durant, les quatre enfants se sont unis dans la crainte du père et la solidarité contre celui qu'ils appréhendent comme une figure tutélaire et tyrannique régnant sur le labyrinthe pragois aux rues étroites, aux hautes murailles et aux sombres cours intérieures. Ils n'ont nul espoir de pouvoir échapper à sa voix tonitruante, à ses colères, à ses critiques incessantes et à son corps écrasant :

J'étais déjà écrasé par la simple existence de ton corps. Il me souvient, par exemple, que nous nous déshabillions souvent ensemble dans une cabine. Moi, maigre chétif, étroit ; toi, fort, grand, large. Déjà, dans la cabine, je me trouvais lamentable, et non seulement en face de toi, mais en face du monde entier, car tu étais pour moi la mesure de toutes choses [...]. Tes moyens les plus efficace d'éducation orale, ceux du moins qui ne manquaient jamais d'avoir leur effet sur moi, étaient les injures, les menaces, l'ironie, un rire méchant et - chose remarquable - tes lamentations sur toi-même ${ }^{7}$.

19 Dans la Lettre au père, où il pratique ce qu'il appelle dans le Procès l'« atermoiement illimité ", qui consiste non à résoudre ses conflits, mais à les regarder en face avec une extrême lucidité et à les analyser complètement pour ne pas risquer de les méconnaître ou de les fuir, Kafka évoque les conséquences désastreuses de l'éducation reçue de son père. Ce dernier, d'un côté, l'attire par ambition sociale vers une culture étrangère, allemande, de l'autre, le ramène en arrière vers un « fantôme de judaïsme » dans lequel l'enfant ne se reconnaît pas et qui nourrit son sentiment de culpabilité :

Plus tard, adolescent, je ne comprenais pas que toi, avec le fantôme de judaïsme dont tu disposais, tu pusses me reprocher de ne pas faire d'efforts [...] pour développer quelque chose de tout aussi inexistant. Car, pour ce que je pouvais en voir, c'était vraiment une bagatelle, une farce, pas même une farce. Tu allais au temple environ quatre jours par an, tu y étais, à tout le moins, plus proche des indifférents que des convaincus, tu t'acquittais patiemment de la prière comme on accomplit une formalités.

\section{L'éducation comme formation ou déformation?}

Selon la définition traditionnelle, l'éducation est censée former l'enfant et assurer un développement physique et moral harmonieux :

L'éducation [...] est la formation de l'homme par lui-même, guidé par son esprit et ses connaissances. L'éducation est la conduite et la formation consciente et sensée de la vie humaine par l'homme lui-même, selon certains principes et idées précis. L'éducation des enfants et des jeunes gens ne représente qu'un cas particulier au sein de ce cadre général ; mais ce cas particulier est d'autant plus important si l'on part du principe que les jeunes gens, et les enfants dans une plus large mesure encore, ont besoin de l'aide et des instructions d'autres personnes pour apprendre à donner une conduite et une forme consciente et sensée à leur existence en recourant à des principes et des idées précises ${ }^{9}$.

L'effet de l'éducation subie par Kafka est exactement contraire au but traditionnellement fixé : l'épanouissement de l'enfant et du jeune homme, sa prise d'assurance croissante grâce au cadre sécurisant de la famille. Or, les trois scènes de l'enfance les plus marquantes évoquées par Kafka sont toutes traumatiques. La première est le souvenir d'une injustice subie par l'enfant qui, ayant réclamé une nuit de l'eau à son père, s'est vu emporter sur la pawlatsche - un balcon extérieur donnant sur une cour, que l'on trouvait alors fréquemment dans les vieilles demeures viennoises et pragoises - pour le faire taire. La seconde, la confrontation de son corps maigre avec le corps puissant de son père à la piscine. La troisième, la description des repas 
familiaux ponctués d'invectives paternelles l'invitant à manger vite et à grandes bouchées. Dans les trois cas, l'enfant joue le rôle de victime d'un bourreau impitoyable et est condamné à l'expulsion (dans la scène de la pawlatsche), l'humiliation (à la piscine) et au gavage (à table). Plus qu'une formation, c'est une véritable déformation de la vision du monde et des potentialités de l'enfant qui résulte de l'éducation de Franz, si bien résumée par Klaus Wagenbach :

Les objets inanimés paraissaient au jeune Franz tout aussi incertains et problématiques que ses relations avec les personnes de son entourage. Son attitude de défense permanente par rapport au monde l'empêchait de distinguer les contours, les couleurs et ne lui permettait de voir que des instantanés, mais alors avec une extrême clairvoyance. Parallèlement, l'abstraction des ordres paternels le rendaient pédant, parce que ces ordres, ne fût-ce que par leur rareté, exigeaient une analyse et une interprétation précises. [...] Tout ce qui sortait de ce cercle lui était totalement indifférent. [...] Il était totalement sous l'emprise de la hiérarchie paternelle, et son père lui apparaissait finalement comme l'instance suprême, tandis que lui-même était son « esclave ${ }^{10}$.

\section{L'enfance et la jeunesse dans l'œuvre de Kafka}

\section{Figures d'enfants}

Si la jeunesse de Kafka ressemble fort à un envol arrêté ou à une émancipation avortée, celle de ses personnages lui est bien semblable.

L'enfance n'y est jamais légère et insouciante: "la frivolité des enfants est incompréhensible ${ }^{11}$ lit-on dans un de ses récits, où, comme dans le Journal, elle est toujours liée à une sensation d'étouffement et d'oppression :

L'enfant de la concierge, quand celle-ci m'a ouvert la porte. Empaqueté dans un vieux châle de femme, blême, avec un petit visage engourdi et potelé. La nuit, la concierge le transporte ainsi arrangé jusqu'à la porte de la rue ${ }^{12}$.

Ce soir en rentrant chez moi, le petit garçon, ficelé comme un paquet gris, qui courait à côté d'un groupe de gamins ${ }^{13}$.

L'enfant frappe fréquemment par sa laideur. Dans Un célibataire entre deux âges, l'enfant de la femme de ménage est difforme et asthmatique :

C'est le portrait de sa mère ; aucune laideur de la vieille femme n'a été oubliée dans ce visage d'enfant. Il est là, avec ses jambes torses, les mains dans les poches de sa culotte, en train de renifler, parce qu'il a déjà un goitre et éprouve de la difficulté à respirer. [...] Dans cette tête informe, il y a malgré tout des pensées d'enfant ${ }^{14}$.

Dans le chapitre sept du Procès, c'est une petite fille bossue et « déjà corrompue » qui indique à Joseph $\mathrm{K}$ la demeure du peintre Titorelli :

La fillette, une gamine bossue qui avait à peine treize ans, lui donna un petit coup de coude et le regarda en coulisse. Ni sa jeunesse ni son infirmité n'avaient pu la préserver de la plus complète corruption. Elle ne souriait même pas, elle examinait gravement $\mathrm{K}$. d'un regard fixe et provocant ${ }^{15}$.

Dans l'ébauche du Journal intitulée Hans et Amalia ${ }^{16}$, deux enfants sont enfermés par un inconnu, figure emblématique d'un père écrasant auquel ils n'échappent que par ruse. Lorsqu'il est vraiment mis en scène, l'enfant apparaît souvent comme un fantôme impalpable, tel cet enfant qui surgit dans la chambre du célibataire un soir et lui dit, lui reprochant sa mélancolie: «votre nature est la mienne et si, de par ma nature, je me 
conduis aimablement avec vous, vous n'avez pas le droit de vous conduire autrement avec moi $»^{17}$.

L'enfant peut aussi être noyé dans un groupe indistinct et insaisissable. C'est le cas des enfants agités, qui courent sous la lune et s'allongent comme des chiens aux pieds de $\mathrm{M}^{\mathrm{me}}$ Cruster dans Tentation au village : « de temps à autre un enfant levait la tête, mais comme il voyait que tous les autres étaient toujours couchés, il s'allongeait de nouveau, lui aussi ${ }^{18}$, ou des fillettes hystériques dont l'attitude exprime un "mélange de puérilité et de perdition» et que le peintre Titorelli, excédé, qualifie de "petites fripouilles » et « petites horreurs $»^{19}$.

Si l'enfant est parfois décrit, il ne semble jamais pleinement réel, soit que son existence soit compromise, soit qu'il soit condamné, comme le jeune Franz, à n'être qu'un fantôme d'enfant pour ne pas faire trop d'ombre aux adultes.

Pour lutter contre l'enfermement familial, l'enfant rêve souvent d'une liberté qui peut se manifester par un renversement soudain de situation, comme dans cette ébauche du Journal où le repas familial se transforme en une grotesque mascarade :

Un dimanche à l'heure du déjeuner, les parents et leurs grands enfants, un fils et une fille, étaient à table. La mère venait de se lever et plongeait la louche dans la soupière ventrue pour servir la soupe, quand, soudain, toute la table se souleva, la nappe s'envola, les mains posées à plat glissèrent de la table et la soupe, où roulaient des boulettes de lard, se répandit sur les genoux du père ${ }^{20}$.

La transgression de la sanction paternelle peut aussi se manifester par une forme de libération des sens :

Un petit garçon était couché dans la baignoire. C'était la première fois que, réalisant son vieux désir, ni sa mère ni sa bonne n'assistaient à son bain. [...] Il s'était passé rapidement l'éponge sur le corps, puis s'était étendu et jouissait de son immobilité dans l'eau chaude ${ }^{21}$.

31 Enfin, la libération peut se produire par la fuite dans l'imaginaire, comme dans une ébauche où un jeune enfant confronté à des devoirs infaisables reçoit l'aide d'un étranger bienfaisant, figure onirique du grand-père affectueux qui s'installe pour aider son petit-fils :

C'était un devoir très difficile et je craignais de ne pas pouvoir en venir à bout. La soirée était d'ailleurs avancée, je m'étais mis au travail beaucoup trop tard, j'avais joué tout un long après-midi dans la rue, cachant ma négligence à mon père qui, peut-être, aurait pu m'aider, et maintenant, tout le monde dormait et j'étais seul devant mon cahier. «Qui m'aidera maintenant?» dis-je tout bas. « Moi », dit un étranger, il s'assit lentement sur une chaise placée à ma droite ${ }^{22}$.

Dans tous les cas, l'enfance est associée à une période d'enfermement, d'oppression, jamais à une phase de bonheur ou d'innocence. Elle se voit totalement démystifiée et échappe à la conception traditionnelle d'un paradis perdu pour devenir un enfer d'où il faut s'échapper le plus tôt possible.

\section{La jeunesse chez Kafka}

\section{La jeune fille épanouie}

Le mythe de la jeunesse comme épanouissement du corps est essentiellement incarné chez Kafka par des jeunes filles aux formes rondes et pleines. À la fin de la Métamorphose 
, le narrateur évoque ainsi l'épanouissement progressif de la jeune Grete, tandis que son frère Gregor dépérit à vue d'œil, selon un étrange principe de vases communicants :

En regardant parler leur fille qui s'animait de plus en plus, M. et $\mathrm{M}^{\mathrm{me}}$ Samsa remarquèrent presque en même temps que Grete, malgré les crèmes de beauté qui lui avaient fait les joues pâles, s'était considérablement épanouie dans les derniers mois; c'était maintenant une jeune fille aux formes pleines [...]. Et il leur sembla voir dans le geste de leur fille une confirmation de leurs nouveaux rêves, un encouragement à leurs bonnes intentions, quand, au terminus du voyage, la petite se leva la première pour étirer son jeune corps ${ }^{23}$.

Dans le Journal et les récits, l'éducation des jeunes filles engendre de fait une forme de sérénité comparable à celle des sœurs de Kafka. Le passage de l'adolescence à l'âge adulte se fait sans heurts et met les jeunes filles en position de force par rapport aux hommes qu'elles séduisent :

L'éducation des jeunes filles, leur passage à l'état adulte, leur adaptation aux lois du monde ont eu de tout temps une importance particulière à mes yeux. Arrivées à ce stade, elles cessent de fuir désespérément celui qui ne les connaît qu'en passant et aimerait bien, en passant, s'entretenir avec elles ; elles restent déjà un peu en place [...]; on n'est plus obligé de les retenir par tous les moyens, regards, menaces ou pouvoirs de l'amour ${ }^{24}$.

\section{Le jeune homme impuissant}

Si la jeune fille est physiquement et moralement épanouie, le jeune homme est généralement de constitution faible et de santé fragile dans les récits ou esquisses qui ponctuent le Journal. De nombreuses ébauches mettent en scène des adolescents petits et chétifs. Ces jeunes hommes sont souvent soit inadaptés à la cruauté sociale ambiante, soit rejetés par leur famille, dans tous les cas marginalisés et solitaires. Ils sont réduits à trois archétypes : le fils, l'étudiant et le célibataire ascétique.

Dans ses premiers grand récits, Kafka met en scène des figures de fils, du Monde citadin au Verdict, de la Métamorphose à l'Amérique. Tous sont soumis à une autorité paternelle écrasante et subissent les reproches de cette figure inhumaine qui les empêche de s'épanouir. Dans le Monde citadin, écrit en 1911, le père d'Oscar retire sa confiance à son fils et le réduit au silence en lui reprochant sa paresse, sa dissipation, sa méchanceté et sa bêtise. Dans le Verdict, le père malade se transforme brusquement en monstre sadique et, après avoir a priori condamné le futur mariage de son fils avec une «oie répugnante ", le condamne à la noyade. Le fils désespéré se jette alors du haut d'un pont. Dans la Métamorphose, le père de Grégoire Samsa expulse son fils réduit à l'état de vermine en lui lançant une pomme qui entraînera sa mort. Enfin, l'Amérique s'ouvre sur une phrase qui évoque le bannissement du jeune Karl Rossmann, exilé outre-Atlantique parce qu'il a été séduit par une bonne qui « l'a rendu père ». Il est ensuite soumis à la tutelle de son oncle Jacob, qui finit par l'abandonner à son sort après que Karl a tenté de s'émanciper de sa tutelle en séduisant la jeune Klara Pollunder.

La deuxième figure archétypale, que l'on trouve essentiellement dans les ébauches du Journal, est le personnage de l'étudiant Kosel, Rense, Blumfeld ou Liman, doubles littéraires de Kafka lui-même. Ils partagent avec lui une tendance certaine à l'asocialité et au mépris de l'autre qui se manifeste par exemple par une phobie du bruit :

Rense fit quelques pas dans la pénombre du couloir, ouvrit la petite porte dérobée de la salle à manger et dit à la bruyante compagnie qui s'y trouvait, presque sans 
regarder de côté : «Je vous en prie, un peu de silence. J'ai un invité. Je demande quelques égards $»^{25}$.

finalement que continuer à parler de son enfance et sa jeunesse manquées. Pourtant, on ne saurait réduire cette donnée biographique à un simple trauma transposé sous forme de fiction. Si la jeunesse est un thème si important aux yeux de Kafka, c'est surtout parce qu'elle permet d'aborder la problématique plus généralement humaniste et politique de l'éducation et de l'aliénation de l'individu par une instance supérieure à laquelle il finit par s'identifier.

\section{Les méfaits de l'éducation}

41 Dans le Journal, le thème de l'éducation aliénante apparaît comme un leitmotiv, notamment dans les six esquisses consacrées à cette problématique qui débutent toutes par l'affirmation selon laquelle son éducation lui a causé beaucoup de tort ${ }^{28}$.

42 La première esquisse développe l'utopie du petit habitant des ruines, de l'être libre que Kafka aurait été s'il n'avait été livré à l'influence aliénante de ses parents et professeurs :

J'eusse préféré être ce petit habitant des ruines rôti par le soleil qui, à travers les décombres m'eût baigné de tous côtés sur le lierre tiède, même si j'avais été faible au début sous la pression de mes bonnes qualités, qui eussent poussé en moi avec la force de l'ivraie ${ }^{29}$.

43 Dans la deuxième variante sur ce thème, le ton change devient celui du reproche, comparé à un poignard qui atteint la famille, les éducateurs et finalement toute la société et qui se voit amplifié dans la troisième ébauche :

Ce reproche se dirige contre une foule de gens ; [...] Il y a là mes parents, quelques membres de ma famille, plusieurs professeurs, une cuisinière bien précise, quelques jeunes filles rencontrées au cours de danse, quelques familiers qui fréquentaient notre maison autrefois, plusieurs écrivains, un maître nageur, un employé derrière 
son guichet, un inspecteur scolaire [...]. Et c'est devant eux tous que j'exprime mon reproche, c'est là ma manière de les présenter les uns aux autres, mais je ne tolère aucune objection ${ }^{30}$. ton extrêmement lyrique, accentué par la métaphore des corbeaux qui projettent de l'ombre sur leur proie et qui représentent, bien sûr, les parents Kafka, puisque le nom « kavka » signifie « choucas ", « Dohle » en allemand. Mais par la puissance du rêve, le corps est comme libéré du poids familial :

J'aurais dû être ce petit habitant des ruines qui prête l'oreille aux cris des choucas dont les ombres passent sur lui et qui se rafraîchit sous la lune, [...] brûlé par le soleil qui, passant à travers les décombres, m'eût baigné de tous côtés sur ma couche de lierre ${ }^{31}$.

Dans les quatrième et cinquième variantes, le corps mis en scène représente une enveloppe séduisante qui masque la faiblesse intérieure héritée de l'éducation «corruptrice » qui a nourri le mépris de soi et la culpabilité permanente que ressent Kafka depuis son plus jeune âge :

Dans mon apparence extérieure, je suis un être humain comme les autres [...]. Mais quand il me manquerait ici la lèvre supérieure, là le pavillon de l'oreille, ici une côte et là un doigt, quand bien même j'aurais des plaques de calvitie sur le crâne et des marques de petite vérole sur la figure, mon physique ne donnerait point encore une image suffisante de mon imperfection intérieure. Cette imperfection n'est pas innée; elle est, en conséquence, d'autant plus douloureuse à porter [...]. Mais, d'autre part, cette imperfection n'est pas non plus méritée, j'ai subi sa naissance sans en être responsable ${ }^{32}$.

$\mathrm{Au}$ fil de ces ébauches sur l'éducation, le reproche se fait plus violent, le ton plus tragique, comme si ce n'est qu'en l'écrivant que Kafka prenait véritablement conscience de la perte irrévocable de son enfance et de sa jeunesse, objets d'une éternelle nostalgie et vecteurs d'une éternelle malédiction envers ses éducateurs :

Ce que je reproche donc à mes éducateurs, c'est le tort qu'ils auraient pu me causer conformément à leurs intentions, c'est cela que je leur reproche, j'exige qu'ils me rendent l'homme que je suis maintenant et comme ils ne le peuvent pas, je donne, plein de reproches et de rires, un coup de tambour que j'envoie dans l'au-delà ${ }^{33}$.

Le seul remède à un tel gâchis reste, pour Kafka, la lucidité, la conscience, la force de vouloir comprendre ce qui lui a tant manqué :

[Mon] visage [...] n'est nullement noir de désespoir, mais blanc et rouge. Il ne le serait pas si mon éducation avait pénétré en moi aussi profondément qu'elle le voulait. Peut-être ma jeunesse a-t-elle été trop brève pour cela, dans ce cas, je fais à pleins poumons l'éloge de sa brièveté, et bien que j'aie maintenant dépassé la quarantaine. C'est la seule chose qui m'ait permis de garder des forces pour prendre conscience des pertes de ma jeunesse, pour me consoler de ces pertes, pour soulever de tous côtés des reproches contre le passé [...]. Mais toutes ces forces ne sont qu'un résidu de celles que je possédais dans mon enfance et qui m'ont, plus que d'autres, livré aux corrupteurs de la jeunesse ${ }^{34}$.

C'est précisément cette force héritée du triste bilan de son enfance et de sa jeunesse, qui lui permet, grâce à la littérature de dépasser son cas particulier en développant, à l'appui de la littérature et de la politique, sa propre théorie de l'éducation idéale. Dans la troisième des lettres qu'il écrit à Elli Hermann, sa sœur, à l'automne 1921, deux ans après la Lettre au père, Kafka évoque Les Voyages de Gulliver de Jonathan Swift. Il y est raconté dans le sixième chapitre que les habitants de Lilliput confient leurs enfants à 
des foyers d'éducation de l'État où les enfants, dès 20 mois, sont arrachés à l'influence aliénante de leurs parents :

Les parents sont les derniers à qui l'on puisse confier l'éducation de leurs enfants et c'est pourquoi on trouve dans toutes les villes des pensions d'État où les parents [...] doivent obligatoirement faire élever leurs enfants [...]. Des maîtres très expérimentés y préparent les enfants à l'avenir qui convient à leur naissance, mais aussi à leurs capacités et à leurs goûts personnels ${ }^{35}$.

Ils sont alors élevés par des nourrices, des éducateurs ou professeurs et ne peuvent recevoir la visite de leurs parents que deux fois par an. Dès onze ans, ils entrent en apprentissage et seuls les fils de bonne famille restent à l'école jusqu'à quinze ans. Dans les trois dernières années de formation, les principes d'éducation très stricts sont assouplis. Swift ne prône pas d'éducation égalitaire, dont le résultat équivaudrait à une uniformisation sociale et culturelle, mais préconise que seuls les membres de l'élite soient envoyés dans ces instituts. Les paysans et ouvriers peuvent garder leurs enfants à la maison, parce que l'éducation qu'ils leur donnent a moins d'influence sur la vie sociale, selon Swift.

S'appuyant sur cette utopie, Kafka développe alors ses pensées sur l'éducation, distinguant l'éducation parentale de l'éducation véritablement humaniste. L'instinct parental étant purement bestial pour lui, et la famille représentant une simple connexion animale repliée sur elle-même, un " organisme déséquilibré », elle risque de provoquer un « inceste spirituel $»^{36}$. Les mots de Kafka ne sont jamais assez durs quand il s'agit de dénoncer l'excès de pouvoir et l'égoïsme des parents :

Il n'y a pas là la moindre trace d'une éducation véritable, qui consiste à développer dans le calme et un amour désintéressé les qualités d'un être en voie de formation [...]. Quand le père «éduque » [...], il trouve dans l'enfant des choses qu'il détestait déjà dans sa propre personne et dont il n'a pu venir à bout, mais qu'il espère maintenant vaincre à coup sûr, car il croit tenir le faible enfant en son pouvoir plus qu'il ne se tient lui-même, si bien que, dans une rage aveugle, il s'empare de l'être en formation sans attendre son développement [...], ou bien il ne voit dans l'enfant que ce qu'il aime, il s'accroche à ce qu'il aime, il se dégrade jusqu'à en faire l'esclave, il le dévore à force d'amour. Tels sont, nés de l'égoïsme, les deux moyens d'éducation des parents, tyrannie et esclavage à tous les degrés ${ }^{37}$.

Les enfants doivent donc au plus tôt être retirés à la charge de leurs parents pour pouvoir pleinement s'épanouir et se connaître et pouvoir éprouver envers leurs parents non de la haine ou du ressentiment, comme Kafka et Elli, mais une véritable reconnaissance, au double sens de gratitude et de respect de l'altérité :

Selon Swift, il faut enlever les enfants aux parents ; autrement dit, l'équilibre dont cet « animal familial » a besoin est obtenu par le fait qu'en enlevant les enfants à la famille, on diffère l'équilibre définitif jusqu'au moment où les enfants, indépendants des parents, leur sont égaux en forces physiques et spirituelles, et où le temps est venu de réaliser le véritable équilibre, l'équilibre dans l'amour ${ }^{38}$.

Si le ton de ces affirmations est si ferme, c'est que Kafka s'est, de longue date, passionné pour le problème de l'éducation. Max Brod lui-même atteste du fait qu'il s'est penché sur les méthodes d'éducation modernes proposées par Maria Montessori, célèbre pour son combat pour l'émancipation de la femme, pour la suppression du travail des enfants et le développement des aptitudes innées de l'enfant. Kafka s'est également intéressé au réformateur anarchiste Francisco Ferrer qui, en 1901, fonda à Barcelone la Escuola moderna et, en 1907, la Liga international para la educacion racional de la infancia, à Paris. Kafka a également lu avec attention les écrits du groupe anarchiste membre du 
comité de réflexion constitué à la fin du siècle pour élaborer des programmes d'instruction scolaire. Les membres en étaient entre autres Tolstoï, Kropotkine, Louise Michel, ainsi que des pédagogues dont les frères Reclus, Jean Graves et Domela Nieuwenhuis, dont Kafka partage bien des points de vue en matière d'éducation. Le but majeur du système scolaire envisagé par les anarchistes consiste à développer la personnalité individuelle, à supprimer la discipline, les programmes et la notation et à introduire un enseignement finalisé, pluridisciplinaire et libéral. Selon Nieuwenhuis, il s'agit de rompre avec le principe d'autorité de l'école et de ne pas faire de l'écolier l'esclave d'une discipline imposée d'en haut, mais de stimuler sa créativité et son indépendance. Kafka partage avec cette dernière le refus de l'autoritarisme parental, la certitude que les parents sont les pires éducateurs pour leurs enfants et l'affirmation selon laquelle à l'autorité parentale succède celle du professeur payé par l'État pour domestiquer l'individu et en faire un bon citoyen. Rompre avec cette logique d'asservissement, c'est donc échapper à l'aliénation non seulement familiale, mais surtout culturelle et politique.

53 Si l'on peut à juste titre établir des parallélismes entre Kafka, Swift et les pédagogues anarchistes, il faut toutefois souligner que si le but de Swift était essentiellement pédagogique et si la position des anarchistes allait de pair avec une critique politique du capitalisme et une critique sociale de la bourgeoisie, l'intérêt de Kafka est essentiellement psychologique. Il utilise Swift ou la pensée anarchiste comme une utopie libératrice destinée à lutter contre le principe d'autorité sous toutes ses formes l'autorité paternelle, la norme familiale, le respect de la loi et de l'État - et à lui permettre de passer du statut de fils obéissant au statut de sujet pensant et écrivant.

\section{Conclusion}

54 Si l'enfance et la jeunesse ne semblaient de prime abord apparaître qu'en filigrane dans l'œuvre littéraire de Kafka, elles n'en représentent pas moins un "non-événement » central dans sa vie. À partir de ce manque initial, de cette jeunesse non seulement perdue, mais d'autant plus douloureuse qu'avortée et "corrompue ", se développe toute l'œuvre et la vie de Kafka, qui déclare lui-même qu'il ne se décide qu'à vingt-huit ans à rattraper les vices de son éducation. Il n'aura pourtant jamais d'enfants pour ne pas leur transmettre le poids de souffrances et de culpabilité qu'Hermann, son père, a fait peser sur ses enfants; en revanche, s'il décrit en 1912 la genèse du Verdict comme une véritable naissance, c'est au double sens de naissance de son œuvre et de naissance de lui-même au monde. De plus, comble du paradoxe, bien que ses récits et écrits autobiographiques rompent tous avec le mythe de la jeunesse dorée et gravitent autour de ce thème de la corruption de la jeunesse, Kafka incarne l'éternelle jeunesse. D'abord parce qu'il est mort jeune, à quarante et un ans, mais aussi parce que, paradoxalement, il n'a cessé de mener une vie de jeune adolescent célibataire, ascétique, sportif, végétarien, entré en littérature comme on entre en religion. Si, dans son œuvre, il a démystifié la jeunesse, il en est donc resté un éternel nostalgique et une figure certes contradictoire, mais d'autant plus emblématique. 


\section{NOTES}

1. Gregor von Rezzori in : Helmut Schink, Jugend als Krankheit, OLV-Buchverlag, Linz, 1980, p. 5.

2. Journal, Euvres complètes III, Gallimard (coll. « La Pléiade »), Paris, 1984, janvier 1914, p. 331.

3. Kafka, Lettre à son père, Euvres complètes IV, Gallimard (coll. « La Pléiade »), Paris, 1989, p. 836.

4. Ibid., p. 838-841. Meschugge : terme yiddish signifiant : « fou, insensé ».

5. Ibid., p. 845-846.

6. Ibid., p. 837.

7. Ibid., p. 838-844.

8. Ibid., p. 861.

9. Fritz-Peter Hager, Wesen, Freiheit und Bildung des Menschen. Philosophie und Erziehung in Antike, Aufklärung und Gegenwart, Haupt, Bern, Stuttgart, 1989, p. 12.

10. Klaus Wagenbach, Kafka, Eine Biographie seiner Jugend, 1883-1912, Francke, Bern, 1958, p. 27.

11. Récits, Gallimard (coll. « La Pléiade »), Paris, 1980, p. 641.

12. Journal, Gallimard (coll. « La Pléiade »), Paris, 1984, 27 novembre 1913, p. 602.

13. Ibid., 12 décembre 1913, p. 324.

14. Un célibataire entre deux âges, Récits, op. cit., p. 366.

15. Le Procès, Gallimard (coll. « La Pléiade »), Paris, 1976, p. 385.

16. Hans et Amalia, Récits, op. cit., p. 384-385.

17. Un jour que j'étais malheureux, Récits, op. cit., p. 133.

18. Tentation au village, Récits, op. cit., p. 286.

19. Le Procès, op. cit., p. 387 sq.

20. Journal, op. cit., 26 janvier 1914, p. 260.

21. Ébauche du 28 septembre 1915, Récits, op. cit., p. 383.

22. Récits, op. cit., p. 403.

23. Kafka, La Métamorphose, Récits, op. cit., p. 244.

24. Kafka, Journal, op. cit., p. 171.

25. Journal, 9 mars 1914, in : Récits, op. cit., p. 261.

26. Journal, juin 1914, in : Récits, op. cit., p. 276.

27. Un célibataire entre deux âges, in : Récits, op. cit., p. 355.

28. Récits, op. cit., p. 122.

29. Ibid., p. 122.

30. Ibid., p. 123.

31. Ibid., p. 124.

32. Ibid., p. 124-125.

33. Ibid., p. 129.

34. Ibid., p. 125.

35. Jonathan Swift, Gulliver's travels, Penguin english library, London, 1967, p. 97.

36. Kafka, Lettre à Elli Hermann, été 1921, in : Lettres à sa famille et ses amis, Euvres complètes III, Gallimard (coll. « La Pléiade »), Paris, 1984, p. 1097.

37. Ibid., p. 1098-1100.

38. Ibid., p. 1101. 


\section{RÉSUMÉS}

Si le thème de l'enfance et de la jeunesse n'apparaît qu'en filigrane dans l'œuvre littéraire de Kafka, il constitue un axe de réflexion majeur de ses écrits autobiographiques, notamment le Journal et la Lettre au père, où l'écrivain évoque son enfance "corrompue " par une éducation autoritaire qui a ancré en lui un profond sentiment de culpabilité, de solitude et une attitude défensive et contemplative face à un monde auquel il s'est senti très tôt étranger. Dans ses récits et romans, Kafka rompt totalement avec le mythe de la jeunesse comme âge d'or et libre développement des aptitudes individuelles: les enfants qu'ils représentent frappent par leur laideur et leur mal-être, tandis que les jeunes hommes, méprisés par les jeunes filles qu'ils tentent vainement de séduire, semblent condamnés au célibat et à la solitude.

Si Kafka ne partage pas le culte de la vie intense et de l'énergie juvénile des expressionnistes dont il est contemporain, il partage avec eux une profonde aversion pour le " monde des pères ", qui se cristallise dans ses réflexions sur l'éducation parentale aliénante. Les six ébauches du Journal consacrées à ce thème en 1910, s'articulent autour du thème du reproche fait aux éducateurs, de la nostalgie d'une jeunesse jamais vécue et de l'utopie du petit habitant des ruines, l'être libre et épanoui qu'il aurait pu devenir, libéré de la tutelle parentale. Onze ans après, en 1921, Kafka approfondit ce thème dans une lettre à sa sœur Elli où il s'appuie sur les Voyages de Gulliver de Swift pour développer une deuxième utopie de l'éducation idéale, laissée aux soins d'éducateurs dans le but de garantir à l'enfant un épanouissement harmonieux de ses facultés, leitmotiv des pédagogues anarchistes dont les écrits ont passionné Kafka.

$\mathrm{Si}$, dans son œuvre de fiction, Kafka a démystifié la jeunesse, il s'en est fait le plus ardent défenseur dans ses écrits autobiographiques en développant l'utopie d'une jeunesse réussie et en restant toujours un "fils", sans descendants autres que les œuvres dont il décrit la genèse comme une naissance et qui lui ont permis, sinon de retrouver son enfance perdue, du moins de la surmonter en devenant créateur d'un monde rêvé.

Ist das Thema der Kindheit und der Jugend im literarischen Werk Franz Kafkas nur zwischen den Zeilen zu lesen, so bildet es doch einen Schwerpunkt seiner autobiographischen Werke, und zwar vor allem im Tagebuch und im Brief an den Vater, wo der Autor seine durch eine autoritäre Erziehung verdorbene Kindheit erwähnt, welche in ihm ein tiefes Gefühl der Schuld, der Einsamkeit und eine Fechter- und Beobachterstellung der Welt gegenüber verankert haben, welcher er sehr früh fremd wurde. In seinen Erzählungen und Romanen distanziert sich Kafka völlig vom Mythos der Jugend als eines goldenen Zeitalters und einer Phase der freien Entfaltung der einzelnen Eigenschaften: die Kinder, die hier beschrieben werden, fallen durch ihre Hässlichkeit und ihr Unbehagen auf, während die Jungen, die von den Mädchen verhöhnt werden, welche sie umsonst zu verführen versuchen, zum Junggesellentum und zur Einsamkeit verurteilt scheinen.

Pflegt Kafka nicht den Kult des intensiven Lebens und der jugendlichen Energie der Expressionisten, seiner Zeitgenossen, so teilt er ihren ausgeprägten Haß gegen die « Väterwelt », welcher sich in seinen Überlegungen zur entfremdenden elterlichen Erziehung kristallisiert. Die sechs Entwürfe des Tagebuchs vom Jahre 1910 kreisen um die Frage des Vorwurfs an die Erzieher, um die Sehnsucht nach einer nie erlebten Jugend und um die Utopie des kleinen Ruinenbewohners, des freien und glücklichen Wesens also, das er hätte werden können, wenn er von der elterlichen Vormundschaft befreit worden wäre. Elf Jahre später, 1921, befasst sich Kafka gründlicher mit diesem Thema in einem Brief an seine Schwester Elli, wo er Swifts Gullivers Reisen erwähnt, um eine zweite Utopie der idealen Erziehung zu entwickeln : letztere wird Erziehern überlassen, damit das Kind die harmonische Entfaltung seiner Anlagen genießt, nach dem 
Vorbild der anarchistischen Pädagogen, deren Schriften Kafka fesselten.

Hat Kafka in seinem erzählerischen Werk die Jugend entmythisiert, so wurde er deren eifrigster Verteidiger in seinen autobiographischen Schriften, wo er die Utopie einer gelungenen Jugend entwickelte, und wo er sich selbst als einen « ewigen Sohn » ohne Nachkommen porträtierte. Besser gesagt, sind seine echten Abkömmlinge seine Werke, deren Genese er wie eine regelrechte Geburt beschreibt, und die es ihm erlaubten, wenn auch nicht seine verlorene Kindheit zurückzuerobern, sie doch zu überwinden, indem er zum Schöpfer einer erträumten Welt wurde.

\section{AUTEUR}

FLORENCE BANCAUD

Université de Rouen 